\title{
Adaptive Kalman Filter Approach and Butterworth Filter Technique for ECG Signal Enhancement
}

\author{
Bharati Sharma ${ }^{1,1}$, R. Jenkin Suji ${ }^{1}$ and Amlan Basu ${ }^{1}$ \\ ${ }^{1}$ Department of Electronics and Communication Engineering, ITM University \\ Gwalior, Madhya Pradesh - 474001, INDIA \\ bharatisharma30@gmail.com,sujijenkin@gmail.com, \\ er.basu.amlan@gmail.com
}

\begin{abstract}
About 15 million people alive today have been influenced by coronary illness. This is a major and critical issue in recent days. There are so many people have been lost their lives due to heart attack and other heart related issues. So, early on analysis and proper cure of heart disease is required to minimize the death rate due to heart disease. For better diagnosis we need exact and consistent tools for determine the fitness of human hearts to analysis the disease ahead of time before it makes around an undesirable changes in human body. For heart diagnosis one of the tools is Electrocardiogram (ECG) and the obtained signal is labeled ECG signal. This ECG signal contaminated by an amount of motion artifacts and noisy elements and deduction of these noisy elements from ECG signal must important before the ECG signal could be utilized for illness diagnosis purpose. There are various filter methods available for denoising ECG signal and select the best one on the dependence of performance parameter like signal to noise ratio (SNR) and power spectrum density (PSD).
\end{abstract}

Keywords: Electrocardiogram (ECG), Kalman filter, Butterworth filter, denoising, signal to noise ratio (SNR), power spectrum density (PSD).

\section{Introduction}

Observation of the ECG (Electrocardiogram) has quite some time been utilized as a part of clinical practice. As of late, the relevance area of ECG observation is extending to regions outer the laboratory [1]. Home observing of patients with rest apnea is one of an example of such an area. There are various ECG monitoring technology available, a move in ECG observing applications is occurring. With advancement in sensor innovation such as material and capacitive terminals, sensors are fused in pieces of the incubator have ended up accessible [2].

Another sensor technology brings the solace of the patient is enhancing continuously. While a few years back the patient needed to accommodate itself according to discomforts of the only available technology, but now a day's patient used to new technology for ECG monitoring and goes with a comfortable treatment of heart diseases [3].

Sometimes these ECG monitoring technologies contaminated due to breathing, and mismatching measurement and explanation of the signal's components therefore, noise artifacts generated into acquired ECG signal and corrupt it. Due to certain stress test, the noise artifacts vary unpredictably. Elimination of Interference in the ECG signal by using various filtering method such as, Adaptive filter and Weiner filter are utilized for removal artifacts from ECG signal [4]. An adaptive Wavelet Weiner 
filtering of ECG signals has been proposed with stationary Wavelet Transform (SWT) and Wavelet Filtering method (WF) compared by different thresholding strategies[5]. This work presents an Adaptive Kalman filter and butter worth filter approach for the estimation and denoising ECG signal. These IIR methods will be utilized for approving the proposed Kalman filter approach.

The proposed procedure in this paper is a utilization of the Kalman filter (KF) for the estimation and evacuation of the noise artifacts in ECG signal. The projected IIR filter methods based on the frequency selective components [6]. The state space model is coordinated with Kalman filter so as to approximate the state variables. The proposed technique recommends an appropriate way for estimation of the noise artifacts of an ECG signal, and is contrasted with the IIR filter methods which basis on the performance parameters.

\section{Methodologies}

\subsection{Kalman filter}

An adaptive Kalman Filter (KF) is a recursive prescient filter that depends on the state model and time varying recursive algorithms. An ECG signals complexes that relates from back to back heartbeats are fundamentally the same yet not indistinguishable. However, while the recording of ECG, the signal is defiled due to some noise interference. An adaptive Kalman filter appraises the state of a dynamic system. This dynamic system can be contaminated by noise. The Kalman filter utilizes estimations to enhance the estimated state [7].

The KF method consists of the prediction and correction of states of the system.

$$
\begin{gathered}
\mathrm{X}_{\mathrm{t}+1}=\mathrm{X}_{\mathrm{t}}+\mathrm{V}_{\mathrm{t}} \\
\mathrm{Yt}_{\mathrm{t}+1}=\mathrm{X}_{\mathrm{t}+1}+\mathrm{W}_{\mathrm{t}+1}
\end{gathered}
$$

Where $\mathrm{x}_{\mathrm{t}+1}$ is the state input of the system, $\mathrm{v}_{\mathrm{t}}$ is the process noise of the system, $\mathrm{Y}_{\mathrm{t}+1}$ is the measurement output of the system and $\mathrm{w}_{t+1}$ is the measurement noise such as noise artifacts.

The prediction is an initial work of the Kalman filter. The predict state or prior state is intended by neglecting the noise of the system. In linear case state vector equation can be represented as:-

$$
\begin{gathered}
X(t)=F \cdot x(t)+n(t) \\
X(t)=F \cdot x(t)
\end{gathered}
$$

Where, $\mathrm{F}$ is the dynamic grid and is consistent, state vector $\mathrm{x}(\mathrm{t})$ and dynamic interference $(\mathrm{t})$ of the system.

The genuine predicted state is a linear combination of the primary state $\mathrm{x}^{-}\left(\mathrm{t}_{0}\right)$ From equation (3) \& (4)

$$
\mathrm{x}^{-}(\mathrm{t})=\mathrm{A}_{0}^{\mathrm{t}} \mathrm{x}^{-}\left(\mathrm{t}_{0}\right)
$$

Where, $\mathrm{A}_{0}^{\mathrm{t}}$ is called the conversion matrix, which transform the primary state $\mathrm{x}^{-}\left(\mathrm{t}_{0}\right)$ to its equivalent $x^{-}(t)$ at point $t$. 
Covariance matrix $\mathrm{P}^{-}\left(\mathrm{t}_{\mathrm{i}}\right)$ of the predicted state vector is attained with the law of error transmission,

$$
P^{-}\left(t_{i}\right)=A \cdot P\left(t_{i-1}\right) \cdot A^{T}+Q
$$

Where, covariance matrix of the noise $\mathrm{Q}$ is a utility of time.

In a correction process we obtained the improved predicted state with observation form at time $\mathrm{t}_{\mathrm{i}}$, thus the posteriori state has form,

$$
\mathrm{x}^{+}\left(\mathrm{t}_{\mathrm{i}}\right)=\mathrm{x}^{-}\left(\mathrm{t}_{\mathrm{i}}\right)+\Delta \mathrm{x}\left(\mathrm{t}_{\mathrm{i}}\right)
$$

And covariance matrix,

$\mathrm{P}^{+}\left(\mathrm{t}_{\mathrm{i}}\right)=\mathrm{P}^{-}\left(\mathrm{t}_{\mathrm{i}}\right)+\Delta \mathrm{P}\left(\mathrm{t}_{\mathrm{i}}\right)$

(8)

$$
\begin{gathered}
\Delta \mathrm{x}\left(\mathrm{t}_{\mathrm{i}}\right)=\mathrm{K}\left(\mathrm{t}_{\mathrm{i}}\right) \cdot\left[\mathrm{l}\left(\mathrm{t}_{\mathrm{i}}\right)-\mathrm{-}^{-}\left(\mathrm{t}_{\mathrm{i}}\right)\right] \\
\mathrm{K}(\mathrm{t})=\mathrm{P}^{-} \mathrm{H}^{\mathrm{T}}\left(\mathrm{HP}^{-} \mathrm{H}^{\mathrm{T}}+\mathrm{R}\left(\mathrm{t}_{\mathrm{i}}\right)\right)^{-1}
\end{gathered}
$$

Where, $\mathrm{K}$ is called the gain matrix. The difference $\left[1\left(\mathrm{t}_{\mathrm{i}}\right)-\mathrm{l}^{-}\left(\mathrm{t}_{\mathrm{i}}\right)\right]$ is identified the extent residual. It reproduces the inconsistency between the predicted measurement and actual extent. At the end corrected state is received by,

$$
\mathrm{x}^{+}\left(\mathrm{t}_{\mathrm{i}}\right)=\mathrm{x}^{-}\left(\mathrm{t}_{\mathrm{i}}\right)+\Delta \mathrm{x}\left(\mathrm{t}_{\mathrm{i}}\right)
$$

The Kalman Filter approach uses to predict and remove the noise artifacts from ECG signal. Though, the equation given in (11) is repeated over input signal. Equation (11) is updated for the Kalman filter.

\subsection{Butterworth filter}

Butterworth filters are having an attribute of maximally level recurrence response and no ripples in the pass band. It moves of nears zero in the stop band [8]. Its reaction inclines off directly towards negative infinity on bode plot. For example, other filter types which have non-monotonic swell in the pass band or stop band, these filters are having a monotonically changing size capacity with $\omega$. The initial $2 n-1$ subordinates for the force capacity as for recurrence are zero. Thus it is conceivable to determine the formula for frequency response,

$$
|H(j \omega)|=\frac{1}{\sqrt{1+(\omega+\omega c)^{2 n}}}
$$

\section{Simulations and Result}

The proposed approaches implemented in Matlab version 2009. To study the performance of the planned method numerous standard data sets have been taken from physio.net, including the MIT-BIH database. This ECG signal corrupted with some noise artifacts and corrupted ECG signal passes through filter and get noise free signal. 


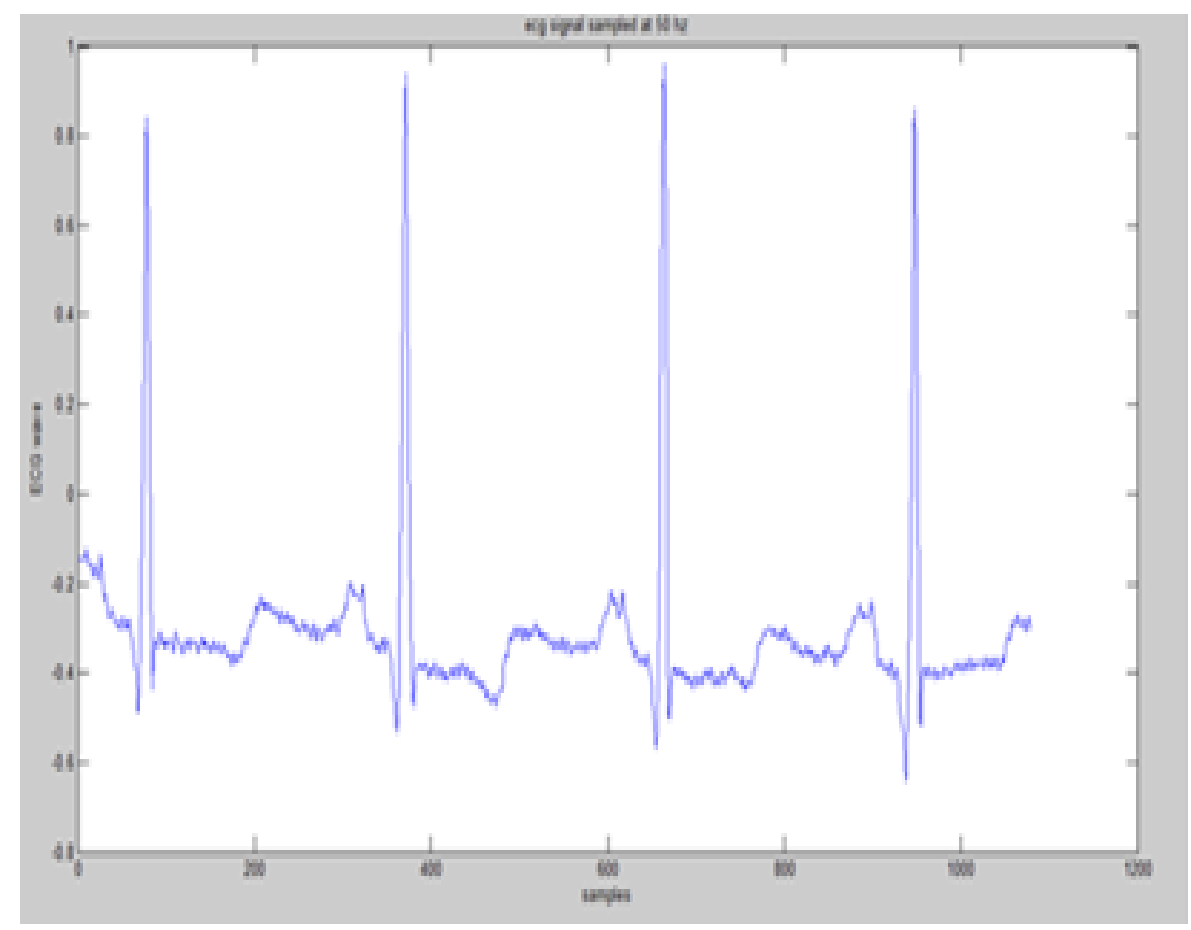

Fig.1 Typical ECG signal

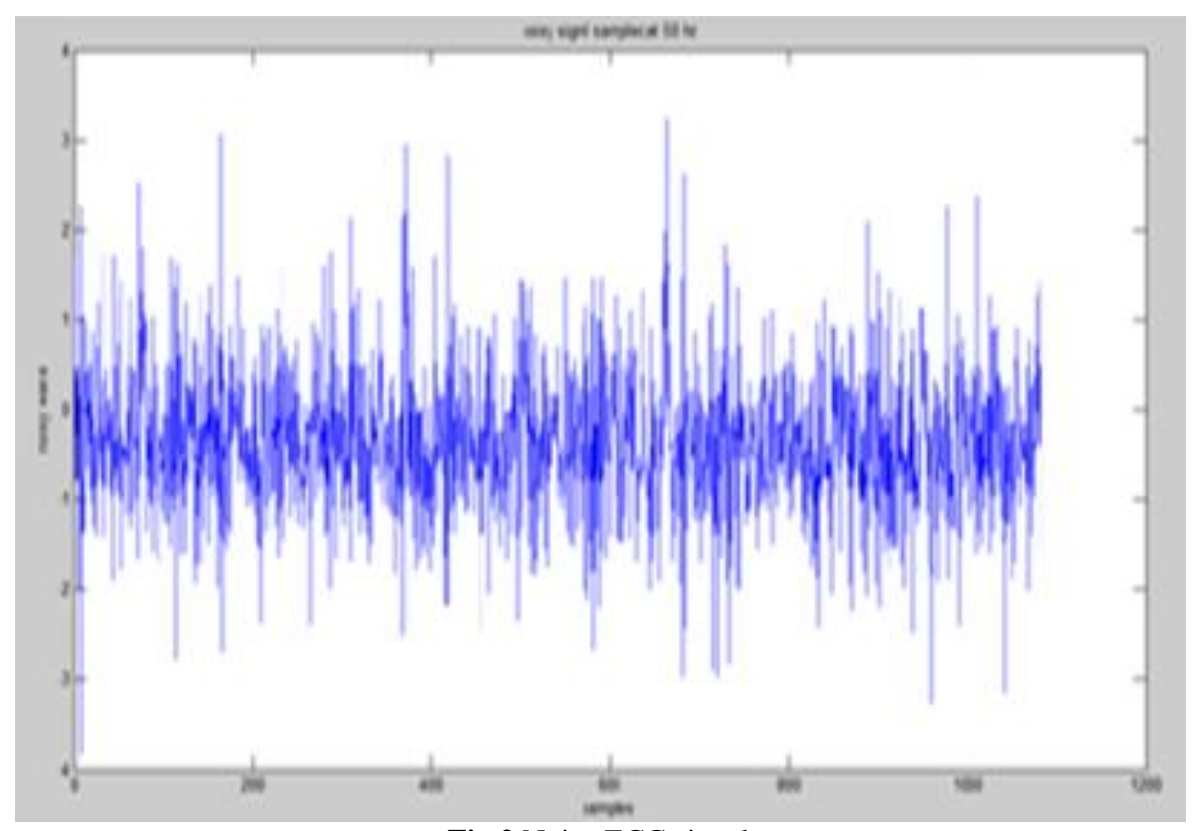

Fig.2 Noisy ECG signal 
a) Graphical representation of Kalman filter method

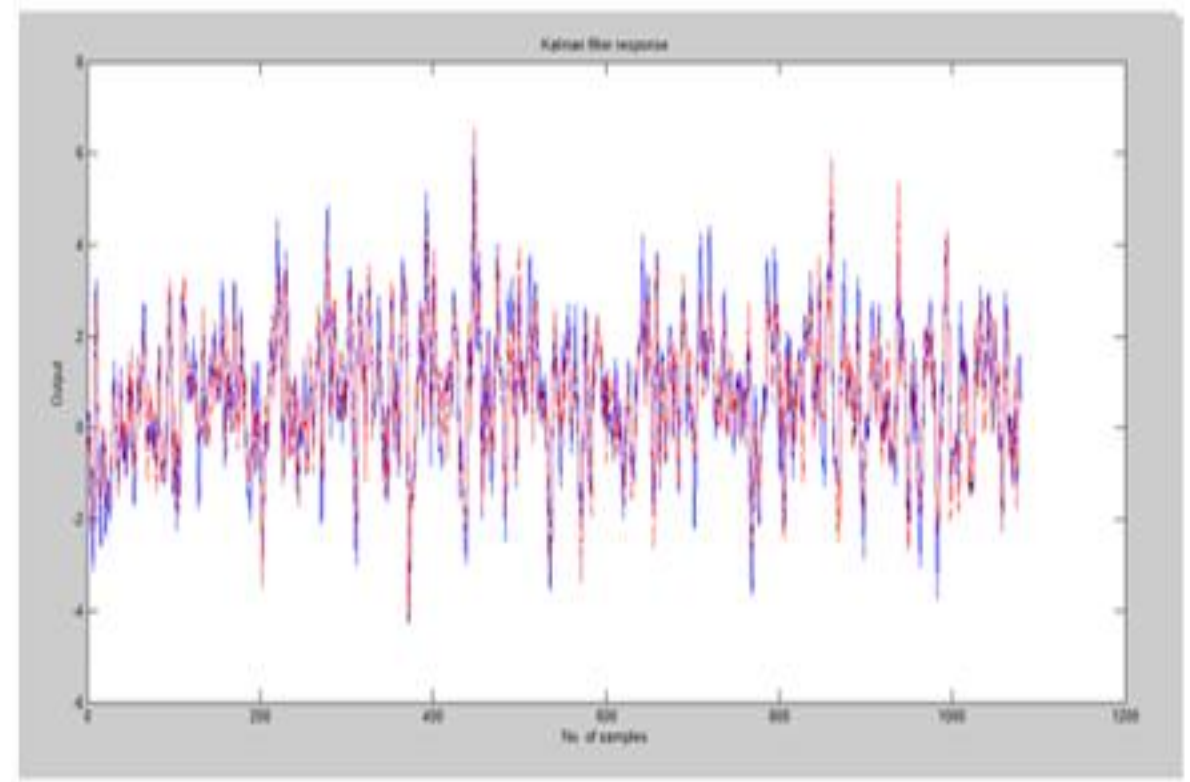

Fig.3 Kalman filter response of ECG signal

Figure 3 illustrates the Kalman filter response of ECG signal and blue line shows the true response of the filter and red shows the filtered response of the signal.

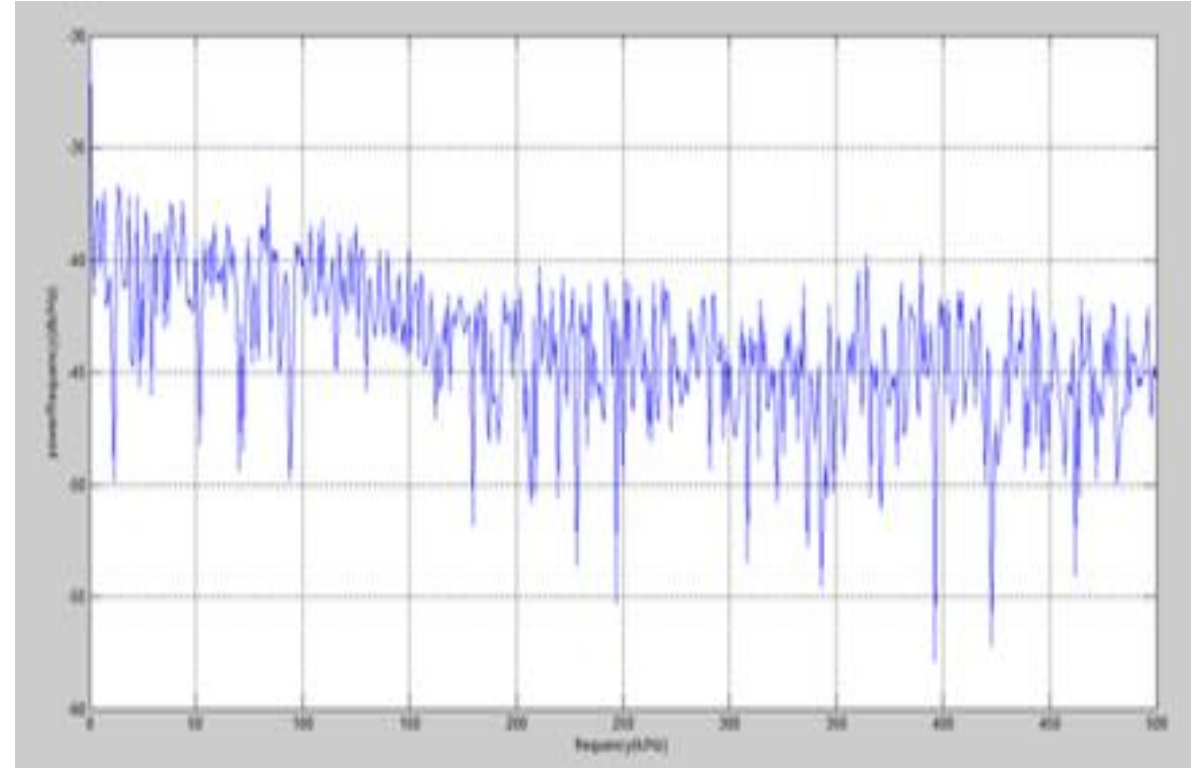

Fig.4 Power spectrum density (PSD) of Kalman filter response 
(b) Graphical representation of Butterworth filter method

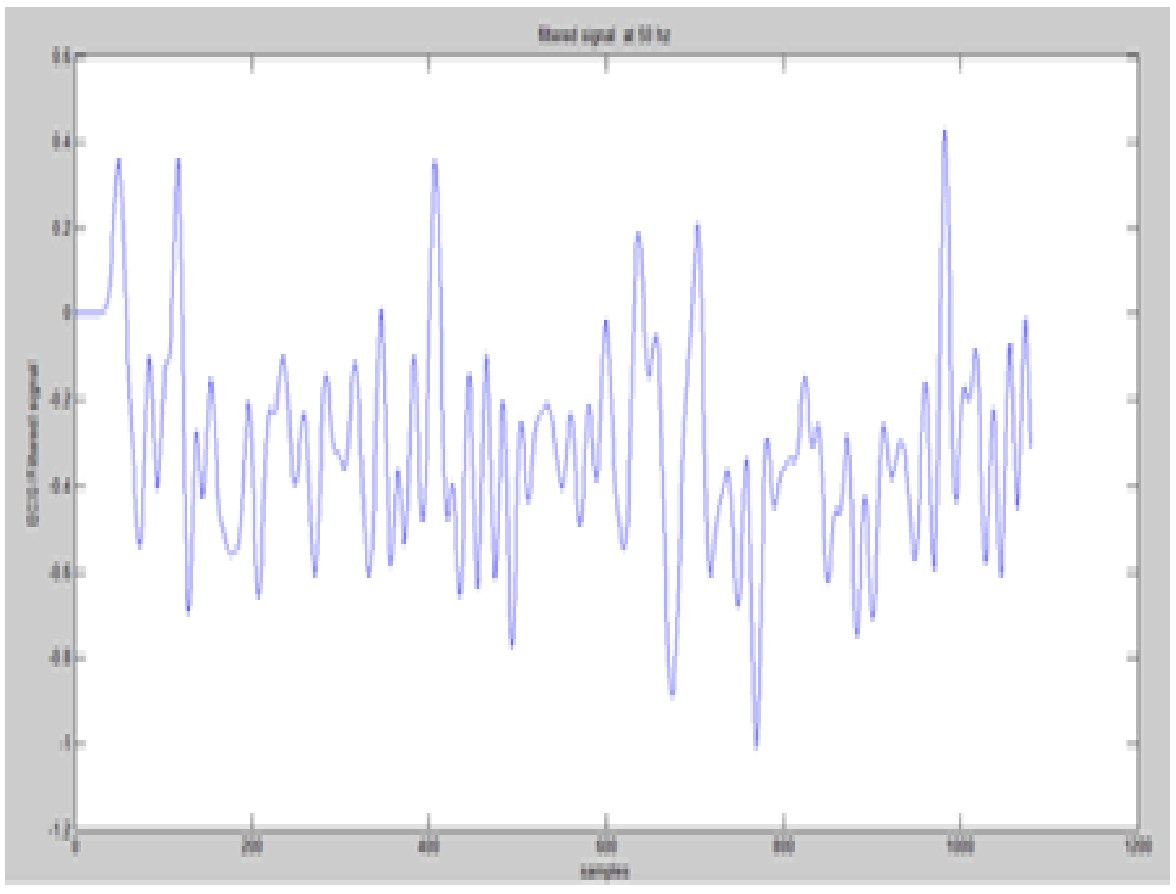

Fig.5 Filtered ECG signal using Butterworth filter

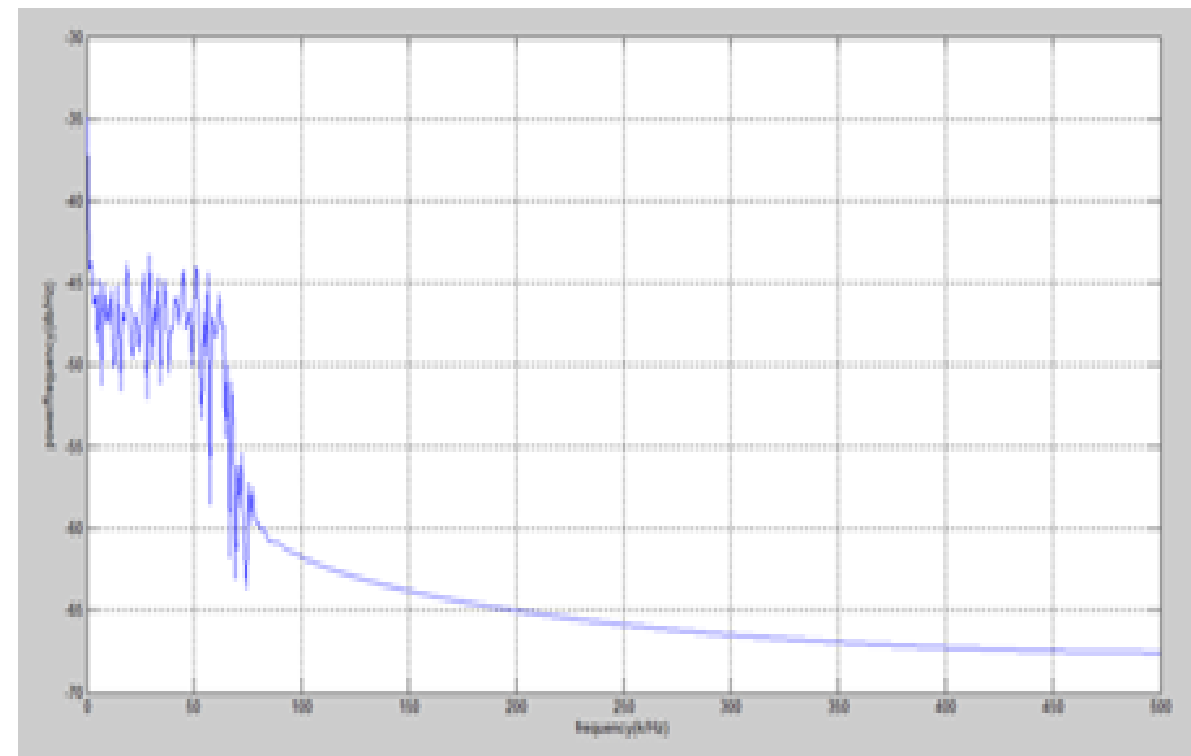

Fig.7 Power spectrum density (PSD) of filter Butterworth signal 
Figure 4 and Figure 7 illustrate the power spectrum density of filtered ECG signal. To compare PSD graph of both the filter and chose best one on the basis of numeric values of graph. The power spectrum density (PSD) and signal to noise ratio (SNR) coming out from distinction between the original signal and signal obtained from each filter methods are given away in Table 1.

Table 1 Comparison between performance parameters of Kalman and Butterworth filter.

\begin{tabular}{|c|c|c|c|}
\hline Parameters & $\begin{array}{l}\text { Original ECG } \\
\text { signal }\end{array}$ & $\begin{array}{ll}\text { Kalman filter } \\
\text { Response }\end{array}$ & $\begin{array}{l}\text { Butterworth filter } \\
\text { Response }\end{array}$ \\
\hline $\begin{array}{l}\text { Signal to ratio } \\
\text { (SNR) }\end{array}$ & -17.4807 & 26.6035 & 5.5091 \\
\hline $\begin{array}{l}\text { Power spectral } \\
\text { density(PSD) }\end{array}$ & -34.5891 & -30.1931 & -34.9085 \\
\hline
\end{tabular}

As can be seen from the table over, the level of contortion is negligible for the Kalman filter technique. The Butterworth filter approach is highly dependent on the filter order and patient's heart rate. Kalman Filter (KF) approach shows better results for denoising ECG signal. It's SNR and PSD is high as compare to Butterworth filter thereby high SNR enhanced the performance of signal as well as PSD of the filtered signal referred to as frequency domain analysis or spectral density estimation. PSD is basically uses for decomposing or denoising a complex signal.

\section{Discussion}

The paper introduced a technique utilized as a part of present time noise artifacts removal. As exposed in the outcomes, the KF approach had insignificant contortion, especially in the QRS complex fragment, when contrasted with the IIR Butterworth filter. The KF approach for the elimination of noise artifacts is best for signal enhancement. Performance parameters like SNR and PSD are increased by Kalman filter approach. High SNR and PSD refers for high stability of the signal So, KF approach has ability to reduce distortion of ECG signal. Butterworth has less SNR and PSD because it is highly dependent on filter order.

\section{Conclusion}

This paper proposed Electrocardiogram denoising execution utilizing Kalman filter and IIR Butterworth filter approach. This Kalman and Butterworth filter method removes noise artifacts and $\mathrm{KF}$ approach has great denoising ability. Simulation Results recover the denoising execution of both Kalman Filter and Butterworth filters are completely different because the performance parameters such as SNR and PSD of Kalman filter has much improved than Butterworth filter. Denoising performance 
and signal enhanced by using Kalman filter approach. For better execution we have chosen Kalman filter rather than Butterworth filter method.

\section{References}

[1] Rik vullings: An Adaptive Kalman Filter for ECG signal Enhancement. IEEE transactions on biomedical engineering, vol. 58, no. 4, pp. 1094 - 1103 (2011)

[2] Mahesh S. Chavan, RA. Agrawal, M.D. Uplane: Suppression of Baseline Wander and Power line interfacing in ECG using Digital IIR filter. International journal of circuits, systems and signal processing, vol. 2 issue 2, pp. 356 - 365 (2008)

[3] Priya Krishnamurthy, N. Swethaanjali, M. Arthi Bala Laxshmi: Comparison of Various Filtering Techniques Used For Removing High Frequency Noise in ECG Signal. International journal of students research in technology \& management, Vol. 3 no. 1, pp. 211-215 (2015)

[4] Nidhi Rastogi, Rajesh Mehra: Analysis of Butterworth and Chebyshev Filters for ECG Denoising Using Wavelets. IOSR Journal of Electronics and Communication Engineering (IOSR-JECE), vol. 6, issue 6, pp. 37 - 44, (2013)

[5] Lukas Smital: Adaptive Wavelet Weiner Filtering of ECG signal. IEEE transactions on biomedical engineering, vol. 60 no.2, pp. 437 - 445 (2013)

[6] Omid Sayadi: ECG Denoising and Compression Using a Modified Extended Kalman Filter Structure. IEEE transaction on biomedical engineering, vol. 55 no. 9, pp. 2240 2248 (2008)

[7] Kohler, B.-U, Henning, C., Orglmeister, R.: The Principles of software $Q R S$ detection. IEEE Engineering in Medicine and Biology Magazine, vol. 21 issue 1, pp. $42-57$ (2002)

[8] Mohan M. Kumar: Simulating Motion Artifacts in ECG using Wiener and Adaptive Filter structure. International journal of emerging technology and advanced engineering, vol. 2 issue 2, pp. $237-260$ (2012) 